\title{
EFEITOS DA ANDIROBA (CARAPA GUIANENSIS) NO EDEMA ARTICULAR E NA DEAMBULAÇÃO DE CAMUNDONGOS COM ARTRITE EXPERIMENTAL INDUZIDA POR ZYMOSAN
}

\author{
EFFECTS OF ANDIROBA (Carapa guianenses) ON JOINT \\ ARTICULATE EDEMA AND AMBULATION OF MICE WITH \\ EXPERIMENTAL ARTHRITIS BY ZYMOZAN INDUCE
}

\author{
Jadson José Guimarães da Poça ${ }^{1}$ \\ Renata Cunha Silva ${ }^{2}$ \\ Emerson Frank Moreira de Souza ${ }^{3}$ \\ Christian Pacheco de Almeida ${ }^{4}$ \\ Tereza Cristina do Reis Ferreira ${ }^{5}$ \\ Alberdan Silva Santos ${ }^{6}$
}

RESUMO: OBJETIVO: Verificar os efeitos da andiroba sobre o edema articular e a deambulação de camundongos induzidos à artrite por Zymosan. MÉTODOS: Os animais foram distribuídos em 4 grupos de 5 camundongos (controle, Zymosan, dexametasona e andiroba), recebendo a administração de acordo com o nome especificado do grupo, uma hora antes da indução à artrite experimental. Seis horas depois, os animais foram reavaliados com o intuito de medir diâmetro articular e o teste de deambulação forçada. RESULTADOS: A média do diâmetro da articulação dos camundongos que receberam tratamento com andiroba $(1,26 \pm 0,61)$ não atingiu resultados esperados, sendo maiores que as médias do grupo controle $(0,296 \pm$ $0,18)$ e dexametasona $(0,22 \pm 0,21)$. Enquanto isso, no teste de deambulação forçada o grupo andiroba $(3,6 \pm 0.55)$ obteve resultados satisfatórios e com scores médios próximos do grupo dexametasona $(3,8 \pm 0.45)$, ou seja, evidenciou-se um baixo comprometimento funcional na deambulação. CONCLUSÃO: Apesar de a andiroba não ter apresentado efeito esperado acerca do edema articular na articulação dos animais induzidas à artrite por Zymosan, em contrapartida, sua administração apresentou efeito satisfatório sobre a deambulação dos animais

\footnotetext{
${ }^{1}$ Graduado em Fisioterapia pela Universidade do Estado do Pará (UEPA).

${ }^{2}$ Graduada em Terapia Ocupacional pela Universidade do Estado do Pará (UEPA).

${ }^{3}$ Acadêmico de Medicina da Universidade Federal do Pará (UFPA).

${ }^{4}$ Graduando em Fisioterapia da Universidade do Estado do Pará (UEPA).

${ }^{5}$ Doutora em Ciências da Reabilitação pela Universidade Nove de Julho (UNINOVE).

${ }^{6}$ Doutor em Bioquímica pela Universidade Federal do Rio de Janeiro (UFRJ).
} 
induzidos a artrite e que receberam dose de andiroba, seus efeitos foram próximo ao da grupo dexametasona, este último um anti-inflamatório de controle positivo. Logo, a andiroba mostrou ser uma alternativa em potencial para tratamento de artrite, necessitando de mais estudos para sua comprovação.

Palavras chave: Artrite Experimental; Camundongos; joelho; inflamação.

ABSTRACT: OBJECTIVE: Verify the effects of andiroba (Carapa guianenses) on joint edema and ambulation of mice with zymozan-induce arthritis. METHODS: The animals were distributed in 4 groups of 5 mice (control, Zymosan, dexamethasone and andiroba), receiving the administration according to the specified name of the group, one hour before induction to experimental arthritis. Six hours later, the animals were reassessed in order to measure joint diameter and the forced ambulation test. RESULTS: The average joint articulation diameter of mice that received treatment with andiroba $(1.26 \pm 0.61)$ didn't reached expected results, being greater than the means of the control group (0.296 \pm 0.18$)$ and dexamethasone $(0.22 \pm 0), 21)$. While, in the forced ambulation test the andiroba group (3.6 \pm 0.55$)$ obtained satisfactory results and with average scores close to the dexamethasone group (3.8 $\pm 0.45)$, that is, evidenced a low functional impairment in ambulation. CONCLUSION: Although andiroba haven't an expected effect on articular edema in the articulation of animals induced by arthritis by Zimosan, in contrast, its administration showed a satisfactory effect on the ambulation of animals induced by arthritis and which received a dose of andiroba, it's effects were close to the dexamethasone group, the latter a positive control anti-inflammatory. Soon, andiroba showed to be a potential alternative for the treatment of arthritis, requiring further studies to prove it.

Keywords: Experimental Arthritis; Mice; Knee; Inflammation. 


\section{INTRODUÇÃO}

A Carapa guianensis é espécie comum da região amazônica, da família das Meliaceae, popularmente conhecida como andiroba. Ela possui um grande destaque na região devido à sua extensa utilização em diversas situações. Seu aproveitamento vai desde suas flores, frutos, sementes até a utilização da madeira. O óleo extraído de sua semente é bastante empregado na medicina popular e tem recebido considerável destaque em pesquisas atuais, nas quais foram constatadas as inúmeras propriedades que a combinação de sua composição química possui, dentre elas: antifúngica, bactericida e antiviral, além de possuir atividade antiinflamatória, minimizando o tal processo por meio da interferência na liberação de mediadores, o que explica a redução dos casos de hiperalgesia e da formação de edema local nesses estudos (OLIVEIRA; HENRIQUES; MACEDO, 2015; VENDRAMIN et al., 2012; MATSUMOTO et al., 2019; PENIDO et al., 2005).

A artrite reumatoide (AR) é uma doença autoimune com etiologia ainda não esclarecida, caracterizada por diversos sintomas como a dor, rigidez articular e edema, que podem levar à incapacidades físicas permanentes, decorrentes do progressivo dano à cartilagem articular e ao osso. Tudo isso é provocado pela inflamação crônica, atingindo principalmente as articulações das mãos, pés, cotovelo e joelho (PRATT; ISSAC, 2014; MOTA et al., 2011). Para além da extensa inflamação, uma das grandes preocupações emergentes no decorrer do desenvolvimento da doença são as incapacidades motoras. Há, portanto, o exemplo característico da redução na amplitude de movimento e limitações para realizar atividades físicas comuns (MOTA et al., 2011; HOOTMAN; HELMICH, 2007).

Em pesquisas envolvendo animais a artrite induzida por Zymosan é um dos métodos experimentais mais conhecidos, onde mimetiza situações que se assemelham à progressão da artrite. (DIMITROVA et al., 2010). Esse modelo tem como característica principal a rápida ativação do sistema imunológico inato provocando alterações na região induzida com características e sintomas 
semelhantes ao da artrite humana (DIMITROVA et al., 2010; MISHARIN et al., 2012).

Observando-se o grau de incapacidade que a doença traz e os elevados custos demandados para o seu tratamento, é interessante a busca de tratamentos alternativos. Diante disso, este estudo busca verificar os efeitos da andiroba sobre o edema articular e a deambulação de camundongos induzidos à artrite por Zymosan.

\section{MÉTODOS}

Trata-se de um estudo experimental randomizado controlado. Os locais de pesquisa foram o Laboratório de Morfofisiologia Aplicada a Saúde e o Biotério Luiz Carlos de Lima Silveira, ambos presentes na Universidade do Estado do Pará (UEPA). Na pesquisa utilizou-se 20 (vinte) camundongos da espécie Mus musculus, machos, adultos, pesando entre 20 e 30 gramas, provenientes do Biotério do Instituto Evandro Chagas (IEC) de Belém do Pará.

Os animais passaram por um processo de aleatorização com a finalidade de serem distribuídos em 4 grupos cada qual com 5 animais: Grupo Controle $(C=5)$, com animais que receberam injeção intra-articular (i.a) de solução salina estéril; Grupo Zymosan $(Z=5)$, com animais que foram apenas induzidos à artrite com injeção intra-articular (i.a) de Zymosan (Sigma Chemical Company); Grupo Zymosan/dexametasona - "Dex" ( $Z D=5)$, com animais que receberam uma dose de 4 $\mathrm{mg} / \mathrm{Kg}$ intraperitoneal de Dexametasona como um anti-inflamatório controle positivo 1 hora antes da indução à artrite com injeção intra-articular (i.a) de Zymosan; e o Grupo Zymosan/andiroba $(Z A=5)$, com animais que receberam por gavagem a solução de andiroba (100 mg/Kg de andiroba diluído em solução de Tween $20 \%) 1$ hora antes da indução à artrite por injeção intra-articular (i.a) de Zymosan (PENIDO et al. 2006).

Os animais foram previamente adaptados e mantidos no Biotério Luiz Carlos de Lima Silveira da Universidade do Estado do Pará (UEPA), por um período de 15 dias e mantidos em gaiolas coletivas $39 \times 26 \times 17 \mathrm{~cm}$. Os recipientes continham 
maravalhas no fundo, trocadas em dias alternados, eram mantidas com iluminação controlada em um ciclo claro/escuro (12/12 horas), recebiam água e ração ad libitum.

Sobre a indução à inflamação articular, primeiramente, os animais foram anestesiados por meio de uma mistura de ketamina 100 mg/Kg (Hospira, Inc.; Lake Forest, IL, USA) e xylazina $5 \mathrm{mg} / \mathrm{Kg}$ (Lloyd, Inc.; Shenandoah, IA, USA) intraperitoneal (i.p). Em seguida, foi feita uma solução com $30 \mathrm{mg}$ de Zymosan (Sigma Chemical Company, St Louis, MO, USA) dissolvidos em $1 \mathrm{ml}$ de solução salina estéril. Dessa solução, apenas $10 \mu \mathrm{l}$ foram injetadas, intra-articular (i.a), na articulação do joelho direito de cada animal que foi induzido à artrite.

Em relação ao óleo extraído da Carapa guianensis (andiroba), foi fornecido pelo Laboratório de Investigação Sistemática em Biotecnologia e Biodiversidade Molecular da Universidade Federal do Pará (UFPA), campus situado na cidade de Belém do Pará, onde foram concedidas as autorizações de coletas e transportes, assim como o acesso ao patrimônio genético. Seu acesso foi solicitado ao Ministério do Meio Ambiente/Instituto Chico Mendes de Conservação da Biodiversidade/SISBIO (MMA/ICMBio/SISBIO) sob número 33336-2.

Quanto aos aspectos éticos, o trabalho foi conduzido de acordo com os padrões internacionais para manuseio e uso de animais a partir da aprovação do comitê de ética em uso de animais da Universidade do Estado do Pará (CEUAUEPA), sob o parecer de número 16/2019. Ademais, foi cadastrado no Sistema Nacional de Gestão do Patrimônio Genético e do Conhecimento Tradicional Associado (SISGEN), sob o número A502114.

Durante o período entre a administração das soluções de andiroba e dexametasona e a eutanásia dos animais foram avaliadas o perímetro articular do joelho para provável verificação de edema articular, como para verificação do desempenho motor. A análise do edema articular foi realizada pela medida dos diâmetros de cada joelho na região de interlinha articular médio-lateralmente, por meio de um paquímetro digital (Paquímetro Digital Em Aço Inox 150 Mm 6 Com Estojo $316119 \mathrm{Mtx}$ ). As mensurações foram realizadas 1 hora antes (basal) e 6 horas após a indução a artrite por Zymosan em milímetros (mm) (SCHNEIDER et al., 2019). 
A avaliação do desempenho motor foi realizada por meio do teste da Rota Rod (EFF 411, Insight $囚)$. O aparelho consiste em uma caixa de acrílico com cilindro de barra de 8 centímetros de diâmetro, instalada na posição transversal a aproximadamente 20 centímetros de altura do piso do equipamento. É horizontalmente dividida em quatro baias com cerca de $10 \mathrm{~cm}$ de largura. A rotatividade do cilindro se realizava por causa do motor com rotação no sentido horário em diferentes velocidades, medidas em rotações por minuto (rpm). 24 horas antes do início dos testes, os animais foram colocados na barra giratória em velocidade de $16 \mathrm{rpm}$ durante 2 minuto, com o objetivo de aclimatação. No teste principal, os animais foram colocados na velocidade e tempo semelhantes ao da aclimatação, avaliando-se a utilização do membro afetado por meio da análise da deambulação forçada, onde o uso da pata foi graduado por com uma escala numérica decrescente, disposta entre 5 a 1, onde $5=$ uso normal do membro; 4= claudicação leve; 3= claudicação grave; 2= intermitente desuso da pata afetada; $1=$ completo desuso da pata (MONVILLE; TORRES; DUNET, 2006; CASTRO et al., 2006).

As análises estatísticas foram realizadas pelo programa GraphPad Prism 5.0. Os seus resultados e variáveis contínuas foram apresentadas por média \pm SEM. Já os testes, aconteceram mediante a análise de variância Kruskal-Wallis seguido por Comparações Student-Newman-Keuls com significância $p<0,05$.

\section{RESULTADOS}

Quanto a variação do diâmetro da articulação ocasionado pela formação do edema no joelho camundongos após 6 horas (Gráfico 1), verificou-se que a administração de andiroba $(100 \mathrm{mg} / \mathrm{Kg})$ não foi suficiente para inibir o desenvolvimento do edema no local da indução à artrite (Figura 1). Diante dessa assertiva, houve o crescimento do diâmetro após indução em média $(1,26 \pm 0,61$ $\mathrm{mm}$ ) para esse grupo, sendo que o grupo controle $(0,296 \pm 0,18 \mathrm{~mm})$ e dexametasona $(0,22 \pm 0,21 \mathrm{~mm})$ obtiveram médias mais satisfatórias para a variável. 


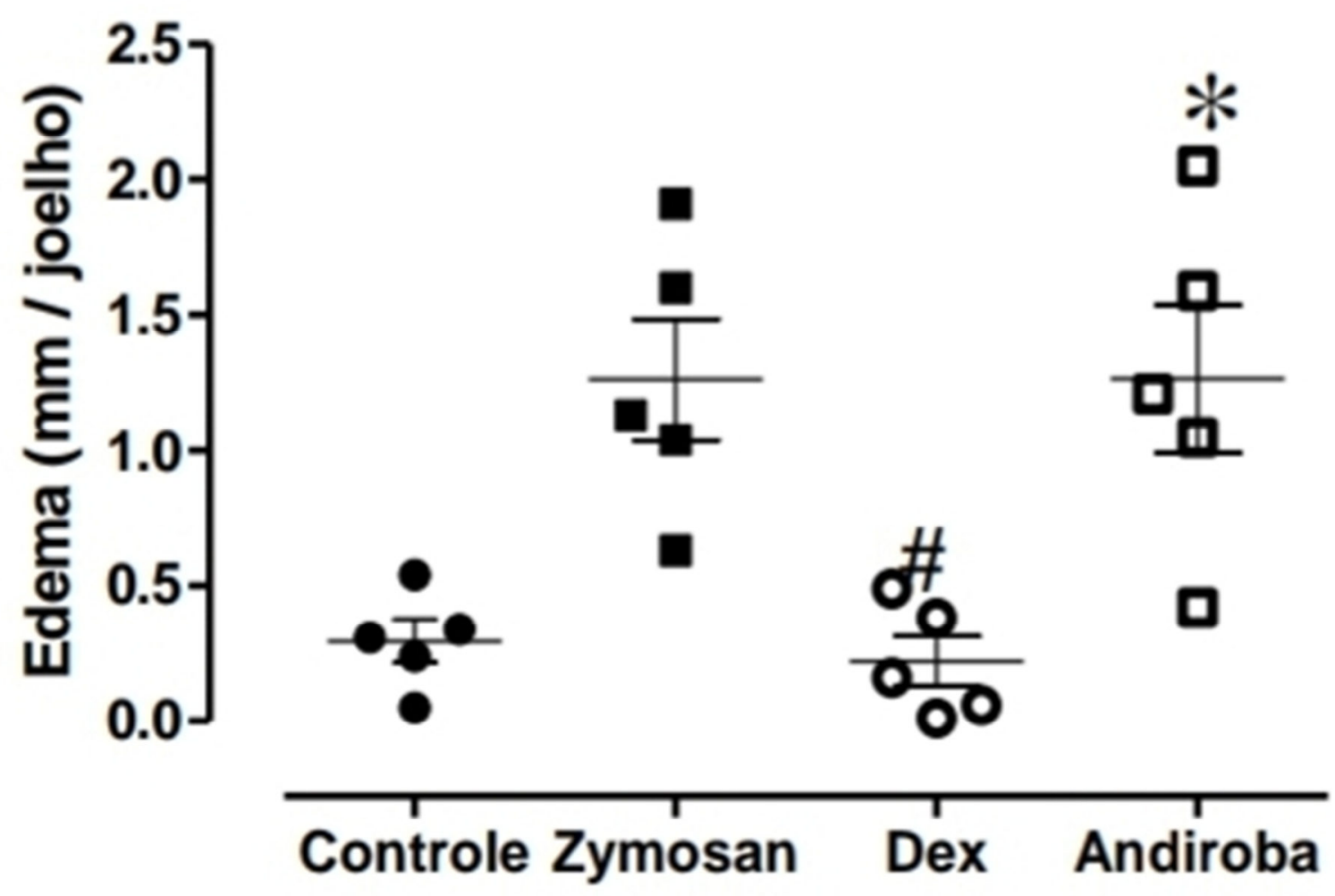

Gráfico 1: Gráfico da variação do diâmetro da articulação do joelho direito do camundongo de acordo com a formação do edema local (em $\mathrm{mm}$ ). Os grupos Zymosan, Dexametasona (Dex) e andiroba foram induzidos à artrite experimental e seis horas depois a articulação foi medida. Os dados são expressos em média \pm SEM ( $n=5$ camundongos por grupo). * $p<0,5$ em comparação ao grupo controle e \# $p<0,5$ em relação ao grupo andiroba. (Analise de variância Kruskal-Wallis seguido por Comparações Student-Newman-Keuls). Fonte: Pesquisadores, 2019.

Diante dos resultados obtidos pelo teste de deambulação forçada (Rota Rod), observou-se que o score do grupo andiroba $(3,6 \pm 0,55)$ resultou em valores do score superior aos do Zymosan $(3 \pm 0.71)$ proporcionando ao primeiro grupo uma menor incapacitação funcional de deambulação após a indução à artrite experimental. Outrossim, notou-se que os valores do score do grupo andiroba foram próximos aos resultados do grupo dexametasona $(3,8 \pm 0.45)$, que é um antiinflamatório de controle positivo no teste, como mostrado no gráfico 2 . 


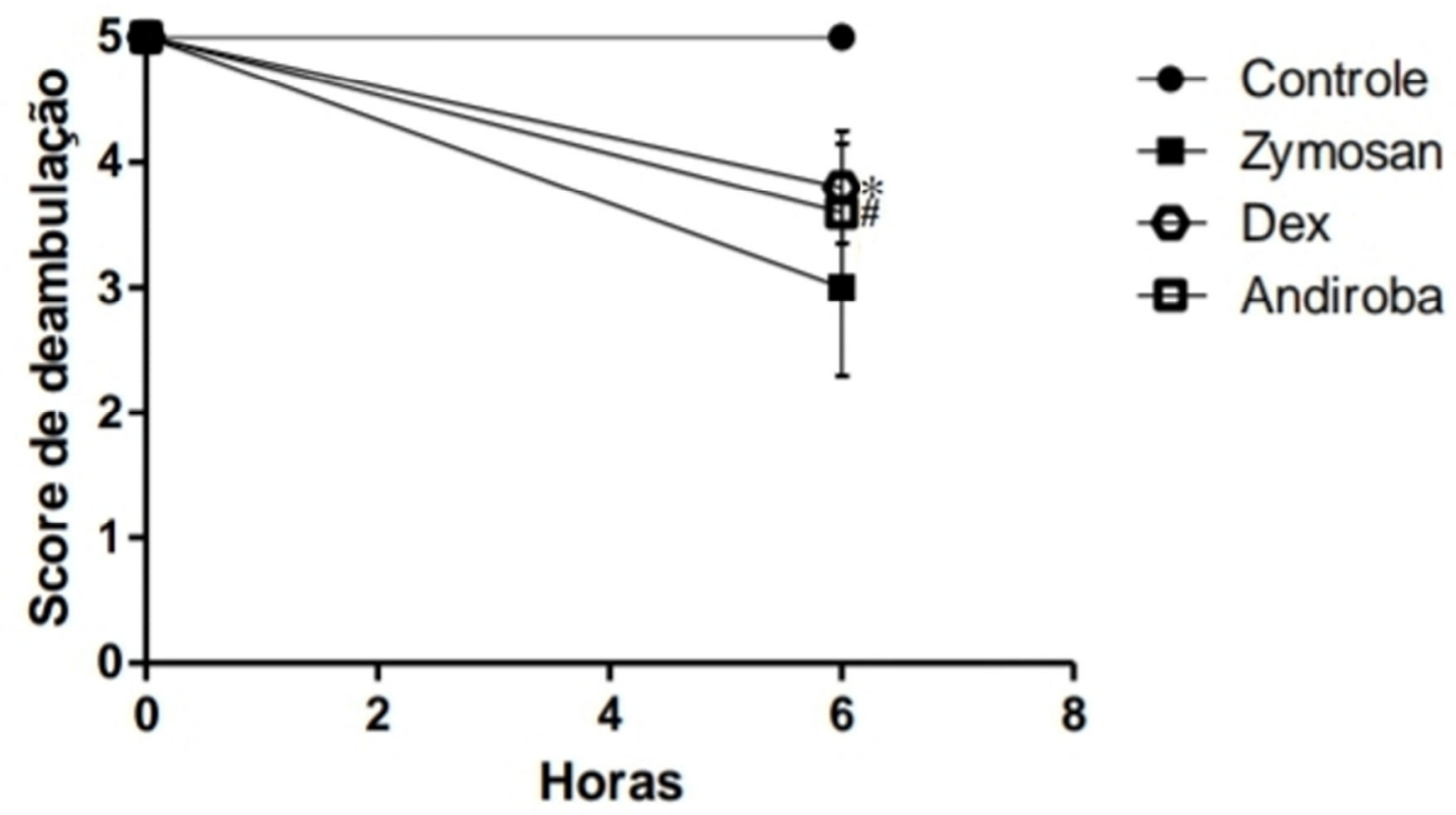

Gráfico 2: Teste de deambulação forçada por meio Rota Rod. O score foi aplicado 6 horas após a indução da artrite. Os dados são expressos em média \pm SEM ( $n=5$ camundongos por grupo). " $p$ e \#p < 0,5 em comparação ao grupo controle. (Análise de variância Kruskal-Wallis seguido por Comparações Student-Newman-Keuls). Fonte: Pesquisadores, 2019.

\section{DISCUSSÃO}

Mesmo não possuindo uma terapia que solucione por completo os sintomas da $A R$, o avanço da medicina sobre a sua amenização tem contribuído para dar melhor qualidade de vida para os pacientes portadores da doença. Terapias complementares e que oferecem custos mais baixos tem se destacado nessa nova abordagem e isso se deve principalmente ao propósito de tornar mais acessível o tratamento a mais pessoas (COMELLA et al., 2016). A andiroba entra nesse cenário devido ao grande potencial anti-inflamatório já mostrado em outras pesquisas (CHIA et al., 2018; COSTA-SILVA et al., 2008). 
De acordo com o estudo de Penido et al. (2006), um estudo experimental realizado em camundongos, a administração da andiroba $(100 \mathrm{mg} / \mathrm{Kg})$ inibiu os efeitos do Zymosan sobre a formação do edema na região da atingida pelo processo instigante da AR. Contudo, neste trabalho, não houve o mesmo resultado significativo semelhante ao estudo que foi tomado como base.

Em relação aos outros grupos, Zymosan e o Dexametasona, os resultados se mostraram semelhantes aos de Formagio-Neto et. al (2019) e Ganova et. al (2017). $\mathrm{Na}$ apuração feita a respeito do grupo Zymosan, houve aumento do perímetro articular e da incapacidade funcional, sintomas característicos da AR. O grupo Dexametasona confirmou a eficácia do fármaco como anti-inflamatório de controle positivo. A afirmativa é possível, afinal, ambos os estudos citados demonstraram a satisfatória função em minimizar os efeitos da artrite induzida por Zymosan, em diversos momentos.

No teste de deambulação, para qual se obteve um resultado expressivo sobre o grupo controle e andiroba, assim como valores significativos entre o grupo andiroba e dexametasona com variações próximas entre esses dois últimos grupos, pode-se inferir que a administração da andiroba contribuiu para o fato de não alterar a capacidade do animal em deambular, mas também de acordo com a estatística os seus efeitos podem ser semelhantes ao da dexametasona. Pode-se supor, à luz das inferências que, apesar dos valores não positivos em relação ao primeiro teste de edema, outros sintomas podem ter sido amenizados, favorecendo para que a deambulação não tivesse sido alterada (MISHARIN et al., 2012).

Segundo Penido et al. (2005), a administração de andiroba reduz a resposta a hiperálgica decorrente da artrite induzida por Zymosan, inferindo sobre um controle considerado sobre a dor. Esse sintoma em questão é um dos principais limitantes sobre a realização de atividades de vida diária, além de contribuir para a diminuição da resistência, da força muscular e do equilíbrio em pessoas que possuem doenças osteoarticulares, entre elas a AR (MAZO et al., 2012). Essa hipótese poderia ser validada com testes que avaliassem o processo álgico durante a indução a artrite assim como durante o tratamento, o que no presente estudo não foi realizado. 


\section{CONCLUSÃO}

De acordo com os resultados apresentados no estudo, observa-se o potencial da andiroba sobre amenização de sintomas decorrentes do desenvolvimento da AR. É importante lembrar que a cura total da doença ainda é um caminho distante, porém o uso de terapias alternativas a fim de amenizar os seus sintomas devem ser sempre levadas em consideração na realização das pesquisas.

A síntese dos achados no estudo traz resultados satisfatórios sobre o score de deambulação do grupo andiroba, isto é, vai de acordo com efeitos fitoterápicos da andiroba já observados em outros estudos. No entanto, sugere-se a realização de futuros estudos voltados a essa questão do binômio andiroba-artrite, procurando reforçar o real potencial da Carapa guianenses na artrite reumatoide.

\section{DECLARAÇÃO DE CONFLITO DE INTERESSE}

Os autores declaram que, no presente estudo, não houve qualquer conflito de interesse. 


\section{REFERÊNCIAS BIBLIOGRÁFICAS}

CASTRO, R. R. et al. A quantitative approach to measure joint pain in experimental osteoarthriti - evidence of a role for nitric oxide. Osteoarthritis and cartilage. v. 14, n.8, p. 769-776, 2006. Disponível em: https://pubmed.ncbi.nlm.nih.gov/16580848/. Acesso em: 08 de novembro de 2019.

CHIA, C. Y. et al. Healing effect of andiroba-based emulsion in cutaneous wound healing via modulation of inflammation and transforming growth factor beta 3. Acta Cirúrgica Brasileira.
v.33,
n.11,
p.1000-1015,
2018.
Disponível
em:

https://www.scielo.br/scielo.php?script=sci_abstract\&pid=S0102-

86502018001101000\&Ing=en\&nrm=iso. Acesso em: 09 de novembro de 2019.

COMELLA, N. F. L., MATILLA M. F., CUESTA, J. A. C. Have complementary therapies demonstrated effectiveness in rheumatoid arthritis? Reumatologia Clínica. v.12, n.3, p.151157, $2016 . \quad$ Disponível em: https://www.sciencedirect.com/science/article/pii/S2173574316000435. Acesso em: 27 de setembro de 2019.

COSTA-SILVA, J. H. et al. Acute and subacute toxicity of the Carapa guianensis Aublet (Meliaceae) seed oil. Journal of Ethnopharmacology. v.116, n.3, p.495-500, 2008. Disponível em: https://pubmed.ncbi.nlm.nih.gov/18281172/. Acesso em: 19 de outubro de 2019.

DIMITROVA P. et al. The role of properdin in murine zymosan-induced arthritis. Molecular immunology. v. 47, p.7-8, 1458-1466, 2010. https://pubmed.ncbi.nlm.nih.gov/20226532/. Acesso em: 15 de setembro de 2019.

FORMAGIO-NETO, F. Anti-inflammatory action of an alkaloid, fraction and extract from Alchornea glandulosa in mice. Journal of ethnopharmacology. v.231, p.66-72, 2019. Disponivel em: https://www.sciencedirect.com/science/article/abs/pii/S0378874118312133. Acesso em: 09 de novembro de 2019.

GANOVA, P. Functional complemente activity is decisive for the development of chronic synovitis, osteophyte formation and processes of cell senescence in zymosan-induced arthritis. Immunology letters. v.190, p.213-220, 2017. Disponível em https://pubmed.ncbi.nlm.nih.gov/28860038/. Acesso em: 10 de setembro de 2019.

HOOTMAN, J. M., HELMICK, C. G. Projections of US prevalence of arthritis and associated activity limitations. Arthritis and Rheumatism. v.54, n.1, p.226-229, 2006. Disponível em: https://pubmed.ncbi.nlm.nih.gov/16385518/. Acesso em: 5 de outubro de 2019.

MATSUMOTO, C. et al. A Limonoid, 7-Deacetoxy-7-Oxogedunin (CG-1) from Andiroba (Carapa guianensis, Meliaceae) Lowers the Accumulation of Intracellular Lipids in Adipocytes via Suppression of IRS-1/Akt-Mediated Glucose Uptake and a Decrease in GLUT4 Expression.. Molecules. 2019 Apr 28; $24 \quad$ (9): 1668. Disponível em: https://pesquisa.bvsalud.org/portal/resource/pt/mdl-31035366. Acesso em 28 de outubro de 2019

MAZO G. Z. et al. Aptidão física, exercícios físicos e doenças osteoarticulares em idosos. Rev Bras Ativ Fis Saúde. v.17, n.4, p.300-306, 2012. Disponível em: https://rbafs.org.br/RBAFS/article/view/1882. Acesso em: 07 de novembro de 2019.

MENDONÇA, A. P; FERRAZ, E I. D. K. Óleo de andiroba: processo tradicional da extração, uso e aspectos sociais no estado do Amazonas. Acta Amazonica, v.37, n.3, p.353-364, 2007. Disponível em: https://www.scielo.br/scielo.php?script=sci_arttext\&pid=S0044- 
59672007000300006. Acesso em 12 de outubro de 2019.

MISHARIN, A. V. et al. Development of a new humanized mouse model to study acute inflammatory arthritis. Journal of translational medicine. v.10, n.1, p.190, 2012. Disponível em: https://pubmed.ncbi.nlm.nih.gov/22974474/. Acesso em: 28 de setembro de 2019.

MONVILLE, C., TORRES E. M., DUNNETT S. B. Comparison of incremental and accelerating protocols of the rotarod test for the assessment of motor deficits in the 6-OHDA model. Journal of neuroscience methods. v.158, n.2, p.219-223, 2006. Disponível em: https://pubmed.ncbi.nlm.nih.gov/16837051/. Acesso em 19 de outubro de 2019.

MOTA, L. M. H et al. Consenso da Sociedade Brasileira de Reumatologia 2011 para o diagnóstico e avaliação inicial da artrite reumatoide. Revista Brasileira de Reumatologia. v.51, n.3, p.207-19, 2011. Disponível em: https://www.scielo.br/scielo.php?script=sci_arttext\&pid=S0482-50042011000300002. Acesso em: 23 de setembro de 2019.

OLIVEIRA, L. L., HENRIQUES A. B., MACEDO, A. F. The effect of growth regulators on two different in vitro- cultured explants of Carapa guianensis. Journal of Medicinal Plants Research. v.9, n.6, p. 169-178, 2015. Disponível em: https://pdfs.semanticscholar.org/3d7c/df54675d33e24a5a8ae62144aab5580457d1.pdf. Acesso em: 08 de setembro de 2019.

PENIDO, $\mathrm{C}$ et al. Antiinflammatory effects of natural tetranortriterpenoids isolated from Carapa guianensis Aublet on zymosan-induced arthritis in mice. Inflammation Research. v. 55, n.11, p.457-464, 2006. Disponível em: https://pubmed.ncbi.nlm.nih.gov/17122962/. Acesso em: 12 de setembro de 2019.

PENIDO, C. et al.. Anti-allergic effects of natural tetranortriterpenoids isolated from Carapa guianensis Aublet on allergen-induced vascular permeability and hyperalgesia. Inflammation Research. v.54, n.7, p.295-303, $2005 . \quad$ Disponível em: https://pubmed.ncbi.nlm.nih.gov/16134059/. Acesso em 12 de outubro de 2019.

PRATT, A. G., ISAACS, J. D. Seronegative rheumatoid arthritis: pathogenetic and therapeutic aspects. Best Practice \& Research Clinical Rheumatology. v.28, n.4, p.651-659, 2014. Disponível em: https://pubmed.ncbi.nlm.nih.gov/25481556/. Acesso em: 07 de novembro de 2019.

SALGADO, H. L. C., SANTOS, A. S. Andiroba oil: a strategic approach to detect antioxidant activity in different lipid groups by TLC. BMC Proceedings, v.8, n.Suppl., p.233, 2014. Disponível em: https://www.ncbi.nlm.nih.gov/pmc/articles/PMC4211088/. Acesso em: 13 de setembro de 2019.

SCHNEIDER, A. H. Estradiol replacement therapy regulates innate immune response in ovariectomized arthritic mice. International immunopharmacology. v.72, p.504-510, 2019. Disponivel em: https://www.sciencedirect.com/science/article/pii/S1567576919306162. Acesso em: 16 de novembro de 2019.

VENDRAMIN, C. R et al. "Cytotoxic efects of andiroba oil (Carapa guianensis) in reproductive system of Rhipicephalus sanguineus (Latreille, 1806) (Acari: Ixodidae) semi-engorged females," Parasitology Research. v.111, n.5, p.1885-1894, 2012. Disponível em: https://pubmed.ncbi.nlm.nih.gov/22797575/. Acesso em: 10 de novembro de 2019. 